\title{
BANKROLL MANAGEMENT IN SIT AND GO POKER TOURNAMENTS
}

\author{
Björn Lantz, \\ PhD, Associate Professor \\ Department of Technology Management and Economics \\ Chalmers University of Technology \\ 41296 Gothenburg \\ SWEDEN \\ E-mail: bjorn.lantz@chalmers.se
}

\begin{abstract}
This study focuses on Sit and go poker tournaments, where a player with an advantage over her opponents needs to manage her bankroll properly. The study applies the Kelly criterion to games that have several outcomes, where the organizer charges a rake. The premise is that an advantage itself is not enough for a poker player to play at any stakes, because risking too large a fraction of the bankroll will result in a negative expected growth rate, even though the game itself is characterized by a positive expected value. Accordingly, this study uses a formula-based approach to address the challenge of identifying games where the player's current bankroll has the highest expected growth rate, while also considering differences in the rake.
\end{abstract}

Keywords: Bankroll management; gambling; Sit and go poker tournaments; Kelly criterion; poker strategy; game theory

\section{INTRODUCTION}

It is well known that the game of poker includes elements of skill as well as elements of luck (Levitt \& Miles, 2014; Croson et al., 2008; Sklansky, 1999). Hence, even though the outcome of a poker game in the short term is determined by chance to a large extent, poker players benefit in the long run from being good at the game. According to Browne (1989), three qualities are required for long-term success in skill-based gambling games such as poker: strong knowledge of the theoretical mechanics of the game, the ability to prevent emotions from negatively affecting play (e.g., to avoid 'tilt'), and the ability to manage the bankroll properly.

There is a lot of scientific literature on the mechanics of poker (e.g., Bowling et al., 2015; Chen \& Ankenman, 2006; Ferguson \& Ferguson, 2003; von Neumann \& Morgenstern, 1944), and some on the emotional aspects of the game (e.g., Palomäki et al., 2014; Palomäki et al., 2013; Hopely and 
Nicki, 2010), but very little on bankroll management, that is, how to determine the right fraction of the bankroll to put at risk in a particular advantageous poker related situation. The only journal article with bankroll management in a poker context as the main issue seems to be Lantz (2015), where the WSOP main event 2014 was analyzed as an example of a large poker tournament with a relatively 'steep' payout structure. In this paper, we look at bankroll management in a Sit and go $(\mathrm{SnG})$ poker tournament context, that is, small tournaments with relatively 'flat' payout structures. Unlike large tournaments with many different payout levels, small tournaments with only a few payout levels can be analyzed more generally and with an explicit formula based approach. To do so is the aim of this paper.

The seasoned long-term winning poker player knows that it makes sense to play at higher stakes than normal when the advantage (i.e., the expected value of the game) is larger than normal ("to take a shot against the fish", as some players would put it). Such a player also knows that the rake level is an important factor in determining her actual advantage in the game. While the rake level rarely appears as an explicit variable in studies on optimal decision making in gambling, the advantage level frequently does (which is a bit of a paradox in that the true rake structure is a known factor in almost all types of games, while the true advantage level is often hard to determine in practice, especially when playing against other human players, as in poker). This paper models the bankroll management problem that advantageous SnG players have to consider, with the rake level as an explicit variable.

In a seminal paper, Kelly (1956) modeled the relation between the advantage the player has in a game and the stakes at which she should play the game. The basic idea is that an advantage player in any type of game is expected to increase her bankroll to a certain amount in the fewest possible bets if she maximizes the expected growth rate of the bankroll. The classical Kelly criterion provides the player with a simple yet elegant formula that shows that in an advantageous game with two possible outcomes (i.e., win $x$ dollars with probability $p$ and lose $y$ dollars with probability $1-p$, where $y(1-p)$ $<x p$ ), the player maximizes the expected growth rate of the bankroll by betting a fraction of her bankroll corresponding to the advantage (i.e. the expected value) divided by the payout of the winning bet. For example, assume that a sports wager that pays 15 to 2 has a $13 \%$ chance of winning. The advantage is then $15 * 0.13-2 *(1-0.13)=0.21$. Thus, a Kelly player would in this situation bet $0.21 / 15=0.014=1.4 \%$ of her current bankroll in order to maximize the expected growth rate of the bankroll. Betting less means that the player does not maximize her opportunity to win money. On the other hand, betting more means that the variance in the expected future bankroll becomes too large in relation to the expected growth. In fact, betting more than $2.88 \%$ of the current bankroll in this example actually leads to a negative expected growth rate of the bankroll because of the elevated risk even though the game is advantageous to the player in terms of a positive expected value. Hence, from an economical point of view, a positive expected value is a necessary but 
not a sufficient condition for participation in a certain poker tournament. A positive expected growth rate of the bankroll is also required.

However, when a game has more than two outcomes, like many types of poker tournaments do, the classical Kelly formula does not work. For example, assume a wager that pays 10 to 1 with a $5 \%$ probability and 2 to 1 with a $20 \%$ probability. The advantage is then $10 * 0.05+2 * 0.20-1 *(1-0.05-$ $0.20)=0.15$, but since there is no unambiguous payout to divide the advantage by, the classical Kelly formula cannot be used to find the optimal fraction of the bankroll to bet. The technical reason is that more outcomes create additional variance in the expected future bankroll. Intuitively, it is easy to understand that the optimal fraction of the bankroll to bet in a single game should be smaller when the risk is higher (other things being equal). But how much smaller?

Recently, Barnett (2011) applied the Kelly criterion when multiple (more than two) outcomes exist in a video poker context. In line with Kelly (1956), Barnett claims that the expected growth rate of the bankroll has a unique maximum, where the first derivative of the expected growth rate of the bankroll equals zero. While this claim certainly is true, Barnett does not provide detailed examples on how players can use it in practice to find the optimal fraction of the bankroll to bet in a single play. In other words, no explicit formula where the player could input odds and probabilities was presented.

The purpose of this paper is to illustrate how the Kelly principle can be used in practice as a bankroll management tool in advantageous games with multiple outcomes in general and in Sit and go poker tournaments in particular, and model the significance of the rake level in a Kelly context. This methodology can be used by advantage players to determine a suitable level at which to play given their current bankroll.

\section{HEADS-UP SNG WITHOUT RAKE}

In a poker tournament context, there are numerous situations where there are exactly two outcomes for any participating player, including heads-up (HU) SnG tournaments, where one player eventually wins the other players' buy-ins, or a satellite tournament, where a small fraction of the field eventually shares the value of the prize pool equally (often a number of seats in a more prestigious tournament).

Assume that an advantage player will win a game with probability $p$ and that the odds of the game are $b$ to 1 (i.e., the player's bankroll will increase by $b$ units if she wins and decrease by 1 unit otherwise). If $x$ is fraction of the bankroll the player bets, the expected growth rate $g(x)$ of the bankroll is $g(x)=(1-p) \log (1-x)+p \log (1+b x)$. The optimal fraction of the bankroll to bet is given by 


$$
\frac{d g(x)}{d x}=\frac{1-p(1+b)+b x}{(x-1)(1+b x)}=0
$$

which simplifies to $x=(p(b+1)-1) / b$, which is the classical Kelly formula (Kelly, 1956). For example, what fraction of her bankroll should a player risk in a SnG tournament with four players where the winner takes all if the probability of winning is $28 \%$ ? The Kelly criterion provides us with the answer: $x=(0.28(3+1)-1) / 3=0.04=4 \%$. In other words, the player should play at stakes where the current bankroll corresponds with $1 / 0.04=$ 25 buy-ins. Hence, if the player's current bankroll is $\$ 500$, she should look for a tournament where the buy-in is $\$ 500 / 25=\$ 20$.

\section{HEADS-UP SNG WITH RAKE}

In a HU SnG tournament without rake, the odds are 1 to 1 by definition, so the game must be characterized by $p>0.5$ in order for the player to have an advantage. If the player also has to pay a rake (i.e., she has to pay a fee to the house on top of her buy-in), $p$ obviously needs to be higher. If the buy-in is denoted by $d$ and the rake percentage is denoted by $r$, the player faces $d$ to $d(1+r)$ odds. Thus, in terms of the normal odds, $b$ to 1 , we have $b=d /(d(1+r))$ $=1 /(1+r)$. The expected growth rate $g(x)$ of the bankroll is now $g(x)=(1-p) \log (1-x)+p \log (1+x(1 /(1+r)))$. The optimal fraction of the bankroll to bet is given by

$$
\frac{d g(x)}{d x}=\frac{1+r-p(r+2)+x}{(x-1)(1+r+x)}=0
$$

which simplifies to $x=p(r+2)-r-1$, which can be used a formula to find the optimal fraction of the bankroll to put at risk. Then, what fraction of her bankroll should a player risk in a HU SnG tournament if the probability of winning is $53 \%$ and the rake level is $5 \%$ ? The formula provides us with the answer: $x=0.53(0.05+2)-0.05-1=0.0365=3.65 \%$. In other words, the player should play at stakes where the current bankroll corresponds with $1 /$ $0.0365 \approx 27$ buy-ins.

The formula is a particularly useful tool when conducting sensitivity analyses. For example, should one play on the same buy-in level when switching to another site with worse players but a higher rake level? Or, how much higher should a player play with her bankroll when the rake level is reduced? For example, if $p=0.51$ and $r=0.05$, the formula shows that the skill advantage does not offset the rake because $x=0.51(0.05+2)-0.05-1=-0.0045=-0.45 \%$, making the game unfavorable for the player. Figure 1 displays, based on the formula, how the 
number of buy-ins $1 / x$ depends on the rake level (for $0.00 \leq r \leq 0.10$ ) when the advantage is $p=0.53$ or $p=0.54$. For example, if $r=0.03$ and $p=0.53$, the player needs a bankroll with 22 buy-ins. It is clear that the rake level has a substantial impact on the required bankroll.

Figure 1: Optimal bankroll size when $p=0.53$ or $p=0.54$

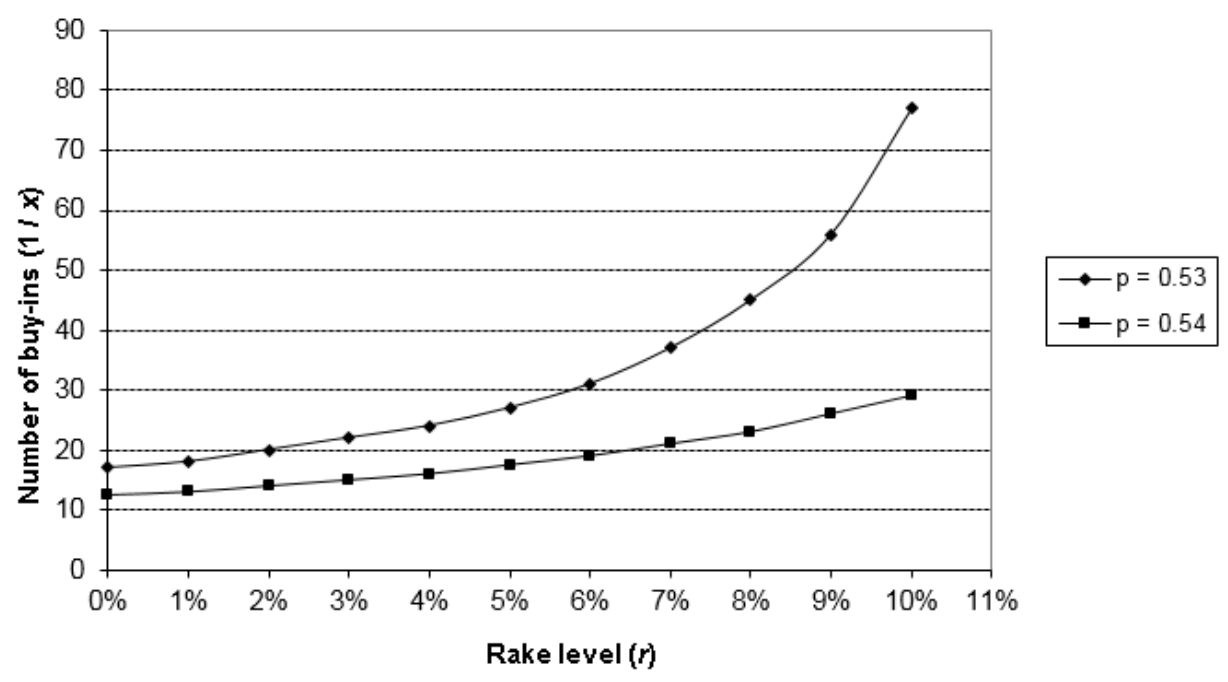

\section{GAMES WITH ASYMMETRIC OUTCOMES AND A RAKE}

If we add the rake factor to a game with $b$ to 1 odds, where $b>1$ (for example, a winner-takes-all SnG tournament with three or more players), our actual odds are $b /(1+r)$ to 1 . The expected growth rate $g(x)$ of the bankroll then becomes $g(x)=(1-p) \log (1-x))+p \log (1+x b /(1+r))$. The optimal fraction of the bankroll to bet is given by

$$
\frac{d g(x)}{d x}=\frac{b x+r+1-p(1+b+r)}{(x-1)(b x+r+1)}=0
$$

which simplifies to the formula $x=(p(b+r+1)-r-1) / b$. Then, for example, what fraction of her bankroll should a player risk in a game with 3 to 1 odds with probability to win $p=30 \%$ and a rake of $5 \%$ ? The above formula provides us with the answer: $x=(0.3(3+0.05+1)-0.05-1) / 3=0.055=$ $5.5 \%$. The formula can be used to perform sensitivity analyses in a similar way as in the previous section. 


\section{GAMES WITH THREE OR MORE OUTCOMES}

When there are three or more outcomes in a game, the classical Kelly formula does not work since it ignores the additional variance compared to a game with only two outcomes. From recommendations regarding bankroll management that can be seen on commercial Internet sites, it seems that $\mathrm{SnG}$ players often use the classical Kelly criterion in their decision making anyway, but based on the average payout level rather than the actual payout levels. For example, www.sitandgoplanet.com currently recommends the formula $x=R^{*} p /(R+1-p)$, where $R$ is the expected return on investment (ROI) and $p$ is the player's probability of finishing in the money (ITM). However, this is an incorrect use of the Kelly criterion.

Assume, for example, that a player plays six-handed $\mathrm{SnG}$ tournaments with two payouts ( $1 / 3$ of the prize pool to second place and $2 / 3$ to the winner) and that she has a $24 \%$ probability of finishing second and a $14 \%$ probability of finishing first. Thus, her bankroll will decrease one buy-in with probability $62 \%$, increase one buy-in with probability $24 \%$, and increase three buy-ins with probability $14 \%$. The expected growth rate of her bankroll then becomes $g(x)=0.62 \log (1-x)+0.24 \log (1+x)+0.14 \log (1+3 x)$. The optimal fraction of the bankroll to bet is given by

$$
\frac{d g(x)}{d x}=\frac{0.24}{1 x+1}+\frac{0.42}{3 x+1}+\frac{0.62}{x-1}=0
$$

which simplifies to $x=0.019$ (since $x$ must be positive). Hence, the player should risk $1.9 \%$ of her bankroll in each game-that is, she should have a bankroll with $1 / 0.019 \approx 53$ buy-ins.

If the above formula from www.sitandgoplanet.com is applied to a game with only two outcomes, we get $x=0.04 * 0.38 /(0.04+1-0.38)=0.023$ since the player's ROI (or advantage) is $R=0.24 * 2+0.14 * 4-1=0.04$ and the probability of finishing ITM is $p=0.24+0.14=0.38$. This corresponds to a bankroll with only $1 / 0.023 \approx 43$ buy-ins. Disregarding the additional variance from the different possible payout levels when the player finishes ITM makes the player risk too high in each game.

The expected growth rate of the bankroll for a game with three or more outcomes, where at least one outcome is negative and at least one outcome is positive, can be written generally as

$$
g(x)=\sum_{i=1}^{m} p_{i} \log \left(1+b_{i} x\right)
$$


where $m$ is the number of outcomes, $p_{i}$ is the probability of outcome $i$, and $b_{i}$ is the profit for outcome $i$. According to Barnett (2011), the optimal fraction of the bankroll to bet is given by

$$
\frac{d g(x)}{d x}=\sum_{i=1}^{m} \frac{b_{i} p_{i}}{1+b_{i} x}=0
$$

However, this theorem cannot be used to derive a closed-form formula for $x$ for the general situation where $m \geq 3$. Each game must be analyzed individually. For example, we can derive a formula for $x$ for a six-handed SnG with two payouts ( $1 / 3$ of the prize pool to second place and $2 / 3$ to the winner). If $p_{2}$ is the probability of finishing second and $p_{1}$ is the probability of winning, the expected growth rate of the bankroll is $g(x)=\left(1-p_{1}-p_{2}\right) \log (1-x)+p_{2} \log (1+x)+p_{1} \log (1+3 x) . \quad$ The optimal fraction of the bankroll to bet is given by

$$
\frac{d g(x)}{d x}=\frac{p_{2}}{1+x}+\frac{3 p_{1}}{1+3 x}-\frac{1-p_{2}-p_{1}}{1-x}=0
$$

which simplifies to $x=$

$\pm\left(\sqrt{9 p_{2}^{2}+12 p_{2} p_{1}-6 p_{2}+4 p_{1}^{2}+4 p_{1}+1}+3 p_{2}+2 p_{1}-2\right) / 3$, where the relevant solution is the smallest positive value of $x$. This formula illustrates the fact that an ROI of, for example, $5 \%$ in this type of tournament says very little about how large the player's bankroll should be.

Table 1: The relations between $p_{1}, p_{2}$, and $\mathrm{x}$ for players with a ROI of $5 \%$

\begin{tabular}{|c|c|c|c|}
\hline$p_{1}$ & $p_{2}$ & $x$ & $1 / x$ \\
\hline 0.000 & 0.525 & 0.0500 & 20 \\
\hline 0.020 & 0.485 & 0.0438 & 23 \\
\hline 0.040 & 0.445 & 0.0389 & 26 \\
\hline 0.060 & 0.405 & 0.0349 & 29 \\
\hline 0.080 & 0.365 & 0.0316 & 32 \\
\hline 0.100 & 0.325 & 0.0288 & 35 \\
\hline 0.120 & 0.285 & 0.0265 & 38 \\
\hline 0.140 & 0.245 & 0.0245 & 41 \\
\hline 0.160 & 0.205 & 0.0227 & 44 \\
\hline 0.180 & 0.165 & 0.0212 & 47 \\
\hline 0.200 & 0.125 & 0.0199 & 50 \\
\hline 0.220 & 0.085 & 0.0188 & 53 \\
\hline 0.240 & 0.045 & 0.0177 & 56 \\
\hline 0.260 & 0.005 & 0.0168 & 60 \\
\hline
\end{tabular}


As Table 1 indicates, an offensive player with a ROI of 5\% who often wins but who also frequently finishes out of the money typically needs a much larger bankroll than a defensive player who often finishes in second place even though she also has an ROI of $5 \%$.

If there is a rake in the above six-handed $\mathrm{SnG}$ tournament, the expected growth rate of the bankroll becomes $g(x)=\left(1-p_{1}-p_{2}\right) \log (1-(1+r) x)+p_{2} \log (1+x)+p_{1} \log (1+3 x)$.

The optimal fraction of the bankroll to bet is then given by

$$
\frac{d g(x)}{d x}=\frac{p_{2}}{1+x}+\frac{3 p_{1}}{1+3 x}+\frac{(1+r)\left(1-p_{2}-p_{1}\right)}{1-(1+r) x}=0
$$

which simplifies to $x=\left(a \pm \sqrt{a^{2}-b}\right) /(6(r+1))$, where

- $\quad a=3 p_{2} r+6 p_{2}+p_{1} r+4 p_{1}+4 r-4$

- $b=4(-3 r-3)\left(p_{2} r+2 p_{2}+p_{1} r+4 p_{1}-r-1\right)$

and the relevant solution is the smallest positive value of $x$. As one might expect, the math quickly becomes messier as the number of outcomes increases. This illustrates the need for good approximations of the Kelly criterion in situations with many outcomes.

\section{CONCLUSION}

There are three factors that work together to determine the optimal bankroll management policy for a $\mathrm{SnG}$ tournament player: the rake level, the value of the different payouts (i.e., how 'steep' the payout structure is), and the probability distribution of the outcomes. A seasoned and long-term winning player knows intuitively that these factors matter, but the exposition in this paper shows how the relations between the factors can be analyzed with an explicit formula approach. The results here can be applicable to poker players' bankroll management in general, and perhaps to conducting sensitivity analyses in particular. They are also applicable to other types of advantageous games with three or more outcomes.

The main difficulty when it comes to the practical application of the ideas discussed here is, of course, the estimation of the probability distribution of the outcome of the tournament. Factors such as the rake level, the number of opponents, and the value of the different payouts are obviously known to all players, but it is rarely possible for a player to know her own true probability to win, to become second, and so on. The typical approach used by many professional players is to use estimates based on previous experience from many similar tournaments, but the problem is that unusually good or 
unusually bad players may have been present to play at specific times. It is still important to have good estimates of the probabilities, however, because, as we have seen in this paper, small errors may result in large differences regarding the optimal fraction of the bankroll to put at risk.

The use of the Kelly principle in bankroll management also highlights the importance of a dynamic approach: that is, the player needs to immediately re-evaluate the stakes at which she should when the size of her bankroll increases or decreases substantially. However, her true advantage may also differ at different stakes - this leads to a kind of circular reasoning given that one obviously has to adapt to the actual levels offered. Given a certain bankroll size, the player's advantage at a certain level may be too large (or small) in terms of the fraction of the bankroll that is actually put at risk, but it may be too small at the next higher level (or too large at the next lower level) for the player to be able to play effectively because her opponents are, on average, better (or worse). Very good players may of course be able to compensate for this effect by using a slightly more defensive (or offensive) strategy without significantly changing their ROI, which, as we have seen, reduces (or increases) the number of buy-ins needed to play at a certain level.

A macro-level interpretation of the analysis in this paper is that poker sites need to adapt their rake strategy when the popularity of poker subsides and mostly better players are still playing. When inferior players drop out, the relative advantage of the remaining profitable players is reduced, and, perhaps more importantly, players who previously had a small advantage become losing players. In order to keep the industry alive, the poker sites will eventually have to lower their rakes to counteract this effect so that a significant fraction of the player collective can expect to make money. The poker industry will hardly survive if only a few players are profitable.

\section{REFERENCES}

Barnett, T. (2011). How Much to Bet on Video Poker. Chance, 24, 10-14.

Bowling, M., Burch, N., Johanson, M. \& Tammelin, O. (2015). Heads-up limit holdem poker is solved. Science, 347-6218, 145-149.

Browne, B. R. (1989). Going on Tilt: Frequent Poker Players and Control. Journal of Gambling Behavior, 5, 3-21.

Chen, B., \& Ankenman, J. (2006). The Mathematics of Poker. Pittsburgh: ConJelCo.

Croson, R., Fishman, P., \& Pope, D. G. (2008). Poker Superstars: Skill or Luck? Chance, 21, 25-28. 
Ferguson, C., \& Ferguson, T. (2003). On the Borel and von Neumann poker models. Game Theory and Applications, 9, 17-32.

Hopely, A., \& Nicki, R. (2010). Predictive factors of excessive online poker playing. Cyberpsychology, Behaviour, and Social Networking, 13, 379385.

Kelly, J. L. (1956). A New Interpretation of Information Rate. The Bell System Technical Journal, July, 917-926.

Lantz, B. (2015). Bankroll management in large poker tournaments. Journal of Gambling Business and Economics, in press.

Levitt, S. D. \& Miles, T. J. (2014). The Role of Skill Versus Luck in Poker: Evidence From the World Series of Poker. Journal of Sports Economics, $15,31-44$.

Palomäki, J., Laakasuo, M., \& Salmela, M. (2013). 'This is just so unfair!': A qualitative analysis of loss-induced emotions and tilting in on-line poker. International Gambling Studies, 13, 255-270.

Palomäki, J., Laakasuo, M., \& Salmela, M. (2014). Losing more by losing it: Poker experience, sensitivity to losses and tilting severity. Journal of Gambling Studies, 30, 187-200.

Sklansky, D. (1999). The Theory of Poker. Las Vegas: Two Plus Two Publishing.

Von Neumann, J., \& Morgenstern, O. (1944). Theory of Games and Economic Behavior. Princeton: Princeton University Press. 